\title{
Article \\ Comparison of the Metastasis Predictive Potential of mRNA and Long Non-Coding RNA Profiling in Systemically Untreated Breast Cancer
}

\author{
Thi T. N. Do ${ }^{1,2}$, Ines Block ${ }^{1,+}$, Mark Burton ${ }^{1,2,3}{ }^{\mathbb{D}}$, Kristina P. Sørensen ${ }^{1}$, Martin J. Larsen ${ }^{1,2}{ }^{\mathbb{D}}$, Martin Bak $^{4,5}$, \\ Søren Cold ${ }^{6}$, Mads Thomassen ${ }^{1,2,3}$, Qihua Tan ${ }^{2,7}$ and Torben A. Kruse ${ }^{1,2,3, *}$
}

check for

updates

Citation: Do, T.T.N.; Block, I.; Burton, M.; Sørensen, K.P.;

Larsen, M.J.; Bak, M.; Cold, S.;

Thomassen, M.; Tan, Q.; Kruse, T.A.

Comparison of the Metastasis

Predictive Potential of mRNA and

Long Non-Coding RNA Profiling in

Systemically Untreated Breast Cancer.

Cancers 2021, 13, 4907. https:/ /

doi.org/10.3390/cancers13194907

Academic Editor: Manny D. Bacolod

Received: 8 May 2021

Accepted: 24 September 2021

Published: 29 September 2021

Publisher's Note: MDPI stays neutral with regard to jurisdictional claims in published maps and institutional affiliations.

Copyright: (C) 2021 by the authors Licensee MDPI, Basel, Switzerland. This article is an open access article distributed under the terms and conditions of the Creative Commons Attribution (CC BY) license (https:/ / creativecommons.org/licenses/by/ $4.0 /)$.
1 Department of Clinical Genetics, Odense University Hospital, 5000 Odense C, Denmark; Nhu.Do@rsyd.dk (T.T.N.D.); Ines.Block@uk-halle.de (I.B.); Mark.Burton@rsyd.dk (M.B.); Kristina.Sorensen1@rsyd.dk (K.P.S.); Martin.Larsen@rsyd.dk (M.J.L.); Mads.Thomassen@rsyd.dk (M.T.)

2 Human Genetics, Department of Clinical Research, University of Southern Denmark, 5000 Odense C, Denmark; Qtan@health.sdu.dk

3 Clinical Genome Center, University of Southern Denmark \& Region of Southern Denmark, 5000 Odense C, Denmark

4 Department of Pathology, Odense University Hospital, 5000 Odense C, Denmark; Martin.Bak@rsyd.dk

5 Department of Pathology, Hospital of Southwest Jutland, 6700 Esbjerg, Denmark

6 Department of Oncology, Odense University Hospital, 5000 Odense C, Denmark; Soeren.Cold@rsyd.dk

7 Epidemiology, Department of Public Health, University of Southern Denmark, 5000 Odense C, Denmark

* Correspondence: Torben.Kruse@rsyd.dk; Tel.: +45-6541-1963

+ Present address: Institute for Physiological Chemistry, Martin Luther University Halle-Wittenberg, 06114 Halle (Saale), Germany.

Simple Summary: To support health care providers in clinical decision-making for breast cancer (BC) patients, profiles of gene activity patterns have previously been developed, where the majority have been based on messenger RNAs (mRNAs), molecules coding for proteins. However, we and others have recently developed profiles based on functional molecules that do not code for proteins-e.g., long non-coding RNAs (lncRNAs) — demonstrating great prognostic potential. Unfortunately, studies comparing such profiles for predicting relapse in BC patients are very scarce. Therefore, we aimed to compare these two types of molecules (mRNAs and lncRNAs) to forecast relapse in low-risk BC patients using advanced machine learning methods with two different approaches. Regardless of approach, our data suggested that profiles based on lncRNAs improved prediction of relapse and demonstrated potential advantages for future profile development.

Abstract: Several gene expression signatures based on mRNAs and a few based on long non-coding RNAs (lncRNAs) have been developed to provide prognostic information beyond clinical evaluation in breast cancer (BC). However, the comparison of such signatures for predicting recurrence is very scarce. Therefore, we compared the prognostic utility of mRNAs and lncRNAs in low-risk BC patients using two different classification strategies. Frozen primary tumor samples from 160 lymph node negative and systemically untreated BC patients were included; 80 developed recurrence-i.e., regional or distant metastasis while 80 remained recurrence-free (mean follow-up of 20.9 years). Patients were pairwise matched for clinicopathological characteristics. Classification based on differential mRNA or lncRNA expression using seven individual machine learning methods and a voting scheme classified patients into risk-subgroups. Classification by the seven methods with a fixed sensitivity of $\geq 90 \%$ resulted in specificities ranging from $16-40 \%$ for mRNA and $38-58 \%$ for lncRNA, and after voting, specificities of $38 \%$ and $60 \%$ respectively. Classifier performance based on an alternative classification approach of balanced accuracy optimization also provided higher specificities for lncRNA than mRNA at comparable sensitivities. Thus, our results suggested that classification followed by voting improved prognostic power using lncRNAs compared to mRNAs regardless of classification strategy. 
Keywords: low-risk breast cancer; lymph node negative; systemically untreated patients; long non-coding RNA; mRNA; prognostic predictors; machine learning methods

\section{Introduction}

In breast cancer (BC), clinical inter-tumor heterogeneity is represented by staging systems, whereas histopathologic and molecular classification reflect morphologic and genetic inter-tumor heterogeneity [1-3]. Intra-tumor heterogeneity also occurs at the genomic, transcriptomic, proteomic, and morphologic level [4-6], causing BC patients to possess varying risk for recurrence development $[7,8]$. For instance, to guide treatment decision, patients are routinely classified based on lymph node status, tumor size, and the positivity or negativity of the tumor for various receptors, such as hormonal receptors or human epidermal growth factor receptor 2 (HER2) (as reviewed by Cardoso et al. [9]).

Due to lack of optimal clinical classification methods, and ability to identify patients with low risk of experiencing recurrence, adjuvant systemic treatments are provided for more than $90 \%$ of all current BC patients as they are classified as high-risk. This is despite the fact that up to $40 \%$ most likely do not benefit from it, as surgical removal of the primary tumor and radiotherapy often are sufficient to prevent recurrence [10,11]. These patients are thus subjected to unnecessary side effects while increasing the expenditures for the health care system. Research focus has, in the past years, shifted from intensifying treatment to also consider reducing overtreatment, as the $\mathrm{BC}$ prognosis due to early diagnosis and advanced treatments has improved over the years. Therefore, it is crucial to predict recurrence at time of diagnosis and determine which patients most likely will never experience recurrence after surgery. By identifying of patients with indolent tumors, we would have the confidence to alleviate the treatments while achieving similar outcomes [12-15].

Since the demonstration of the prognostic role of gene expression profiling in primary breast tumors by van't Veer et al., several mRNA-based signatures have been developed to provide prognostic information beyond clinical evaluation (e.g., MammaPrint, Oncotype DX, PAM50) [16-18]. Despite the focus on mRNAs, gene expression profiles based on long non-coding RNAs (lncRNAs) have recently emerged, demonstrating great prognostic potential in BC [19-21]. LncRNAs have become a research area of intensive focus and their biology and roles in tumor development and progression are widely studied. Associations between several lncRNAs and stage, as well as prognosis of multiple tumor types, have been found and the therapeutic potential of IncRNAs has also been extensively studied (as reviewed by Borkiewicz et al. [22]). It is however extremely difficult to generalize findings from such studies across different tumor types due to distinct biology.

In continuation of this, it is also difficult to generalize biological insights into clinical application. The clinical aspect of lncRNAs as biomarkers for prediction of clinical outcome has been studied to a lesser degree [19-21]. Even more limited are studies comparing the relative ability of mRNA and lncRNA-based signatures to predict recurrence. In this study, we compared mRNA and lncRNA-based signatures using two different classification strategies which furthermore were compared for clinical application. This study is, to the best of our knowledge, the first to compare such signatures in systemically untreated low-risk BC patients with very long follow-up.

\section{Materials and Methods}

\subsection{Tumor Biopsies}

The study included frozen tumor biopsies from lymph node negative (LNN) and systemically untreated low-risk BC patients who were diagnosed from 1980 to 2003 on the island of Funen, Denmark. A total of 160 frozen tumor biopsies were collected as previously described [19]. Half of the patients developed recurrence that is, regional or distant metastasis within 10 years after diagnosis while the remaining half were recurrencefree (mean follow-up of 20.9 years). Patient biopsies were pairwise matched according to 
age (range: 33-88 years), tumor type, tumor diameter (range: $4-50 \mathrm{~mm}$ ), year of surgery, receptor status (ER, PR, n/a), and histological grade (grade 1-3 or n/a) (Table 1).

Table 1. Patient and tumor characteristics.

\begin{tabular}{|c|c|c|}
\hline Characteristics & Recurrence Development & Recurrence-Free \\
\hline No. of patients & $80(50)$ & $80(50)$ \\
\hline \multicolumn{3}{|l|}{$\begin{array}{c}\text { Age at diagnosis } \\
\text { (range: } 33-88 \text { years) }\end{array}$} \\
\hline$\leq 50$ years & $14(8.8)$ & $10(6.3)$ \\
\hline$>50$ years & $66(41.3)$ & $70(43.8)$ \\
\hline \multicolumn{3}{|l|}{ Tumor size } \\
\hline$<2 \mathrm{~cm}$ & $27(16.9)$ & $30(18.8)$ \\
\hline $2-5 \mathrm{~cm}$ & $53(33.1)$ & $49(30.6)$ \\
\hline $\mathrm{n} / \mathrm{a}$ & & $1(0.6)$ \\
\hline \multicolumn{3}{|l|}{ Estrogen receptor status ${ }^{a}$} \\
\hline Positive & $52(32.5)$ & $50(31.3)$ \\
\hline Negative & $22(13.8)$ & $24(15)$ \\
\hline $\mathrm{n} / \mathrm{a}$ & $6(3.8)$ & $6(3.75)$ \\
\hline \multicolumn{3}{|l|}{ Tumor type } \\
\hline Invasive ductal carcinoma (IDC) & $62(38.8)$ & $65(40.6)$ \\
\hline Invasive lobular carcinoma (ILC) & $9(5.6)$ & $9(5.6)$ \\
\hline Mucinous carcinoma & $2(1.3)$ & $2(1.3)$ \\
\hline Papillary carcinoma & $3(1.9)$ & $2(1.3)$ \\
\hline Carcinoma with metaplasia & $2(1.3)$ & $2(1.3)$ \\
\hline $\mathrm{n} / \mathrm{a}$ & $2(1.3)$ & - \\
\hline \multicolumn{3}{|l|}{ Histologic grade } \\
\hline 1 (good) & $12(7.5)$ & $15(9.4)$ \\
\hline 2 (intermediate) & $28(17.5)$ & $25(15.6)$ \\
\hline 3 (poor) & $22(13.8)$ & $24(15)$ \\
\hline $\mathrm{n} / \mathrm{a}$ & $18(11.3)$ & $16(10)$ \\
\hline $\begin{array}{l}\text { Median year of surgery } \\
\quad \text { (range 1980-2003) }\end{array}$ & 1993 & 1994 \\
\hline Mean time to recurrence (months) & 58.5 & $\mathrm{n} / \mathrm{a}$ \\
\hline Mean follow-up (months) & 88.3 & 250.35 \\
\hline Alive at end of follow-up & 1 & 48 \\
\hline
\end{tabular}

n/a: not available/applicable; ${ }^{\text {a }}$ as defined by immunohistochemistry. Values in parentheses indicate percentage of patients in each category out of the whole sample size.

Independence of traditional prognostic markers was achieved by pairwise matching the patients, increasing the power by enriching informative clinical endpoints while bias related to sample retrieval, storage, and diagnostic procedures was reduced. All clinicopathological information used for sample matching was acquired from the Danish Breast Cancer Cooperative Group (DBCG) database, the Funen pathology database, or the nationwide pathology database. The study was approved by the Danish National Committee on Health Research (S-VF-20020142). The study was retrospective, and no informed consent was obtained from the included patients as approved by the Ethical Committee [19]. 


\subsection{Microarray Analysis and Re-Annotation}

Total RNA was extracted from freshly frozen tumor biopsies as previously described [23] A modified standard design of the SurePrint G3 Human GE $8 \times 60 \mathrm{k}$ oligonucleotide slides (G4102A) by Agilent Technologies (Santa Clara, Santa Clara County, CA, USA) was applied for gene expression analysis. The matched sample pairs were kept together during all steps of RNA extraction, hybridization, and gene expression analysis. LncRNA and mRNA expression data have previously been deposited in NCBI's Gene Expression Omnibus [24] and are accessible through GEO Series accession number GSE48408 [19]. To select the probes covering mRNAs and lncRNAs, chromosomal positions of the probes in the annotation file from Agilent were matched to the positions in the Human RNA catalog from GENCODE v. 16 [25] as previously described $[19,26]$.

Gene expression data were analyzed in 160 freshly frozen primary tumors from LNN and systemically untreated low-risk patients with invasive BC. Re-annotation resulted in identification of 21,858 and $4810 \mathrm{mRNA}$ and lncRNA probes on the Agilent array. Further analysis was performed using all mRNA probes and probes covering 2811 unique lncRNAs (26). Comparing the two patient groups, recurrence development vs. recurrence-free using paired $t$-tests identified 148 and 160 differentially expressed lncRNAs and mRNAs (FDR $\leq 0.05$ ). The lists of the differentially expressed RNAs are shown in Tables S1 and S2.

To avoid any bias, the input lists of RNA transcripts for the procedure of outcome prediction using leave-one-pair-out cross-validation (LOPOCV) were applied unmodified, i.e., with 21,858 and $2811 \mathrm{mRNAs}$ and lncRNA probes.

\subsection{Data Processing, Classification and Voting}

Array scanning and data preprocessing were performed as previously described [23]. Seven of the most commonly used machine learning methods, found through literature search on breast cancer classification were applied for sample classification: linear discriminant analysis (LDA), support vector machines based on a radial kernel (R-SVM) or linear kernel (L-SVM), random forest (RF), naïve Bayes (NB), COX risk score (COX-RS), and logistic regression (LR). LOPOCV was applied to provide an unbiased estimate of classifier performance (Figure A1). This approach has proven to be optimal for analysis of smaller datasets [27,28]. We applied a voting scheme for gene expression-based classification of BC patients, which previously has been shown to improve classification performance [29].

All classification procedures and statistical analyses were performed using the R opensource environment (version 4.0.2, https: / / cran.r-project.org/, accessed on 23 June 2020). For SVM and NB based classification, we used the e1071 R-package (https:/ / cran.r-project. org/web / packages/e1071/index.html, accessed on 23 June 2020 for e1071 and the following R-packages), while the RF, COX-RS, LDA, and LR based classification procedures were performed using the randomForest (https:/ / cran.r-project.org/package=randomForest, accessed on 23 June 2020), survival (https:/ / cran.r-project.org / package=survival, accessed on 23 June 2020), MASS (https: / / cran.r-project.org/ package=MASS, accessed on 23 June 2020), and stats (https://stat.ethz.ch/R-manual/R-devel/library/stats/html/stats-package.html, accessed on 23 June 2020) R-packages, respectively. We performed classification according to the differentially expressed RNA molecules with above mentioned machine learning methods, applying two different approaches which: (1) provided $\geq 90 \%$ sensitivity while maximizing specificity; and (2) optimized balanced accuracy (bAcc). Both optimization strategies were followed by voting with a final sensitivity of $90 \%$ (Figure 1). Balanced accuracy is the arithmetic mean of two metrics: sensitivity, which is the proportion of truly affected subjects who are correctly classified as positive; and specificity, which measures the proportion of truly unaffected subjects who are correctly identified as negative [30].

The integrated voting results were obtained using seven distinctive cutoffs ranging from 1-7, representing different degrees of classification agreement between the seven methods in terms of recurrence votes. A cutoff of 1 meant that one or more votes for recurrence, placed the patient as high-risk whereas zero votes classified the patient as 
low-risk, while a cutoff of 7 meant that if all votes were assigned for recurrence, the patient was placed as high-risk, while six or less votes corresponded to low-risk.

A more detailed description of the applied procedures is provided in the Appendix A.

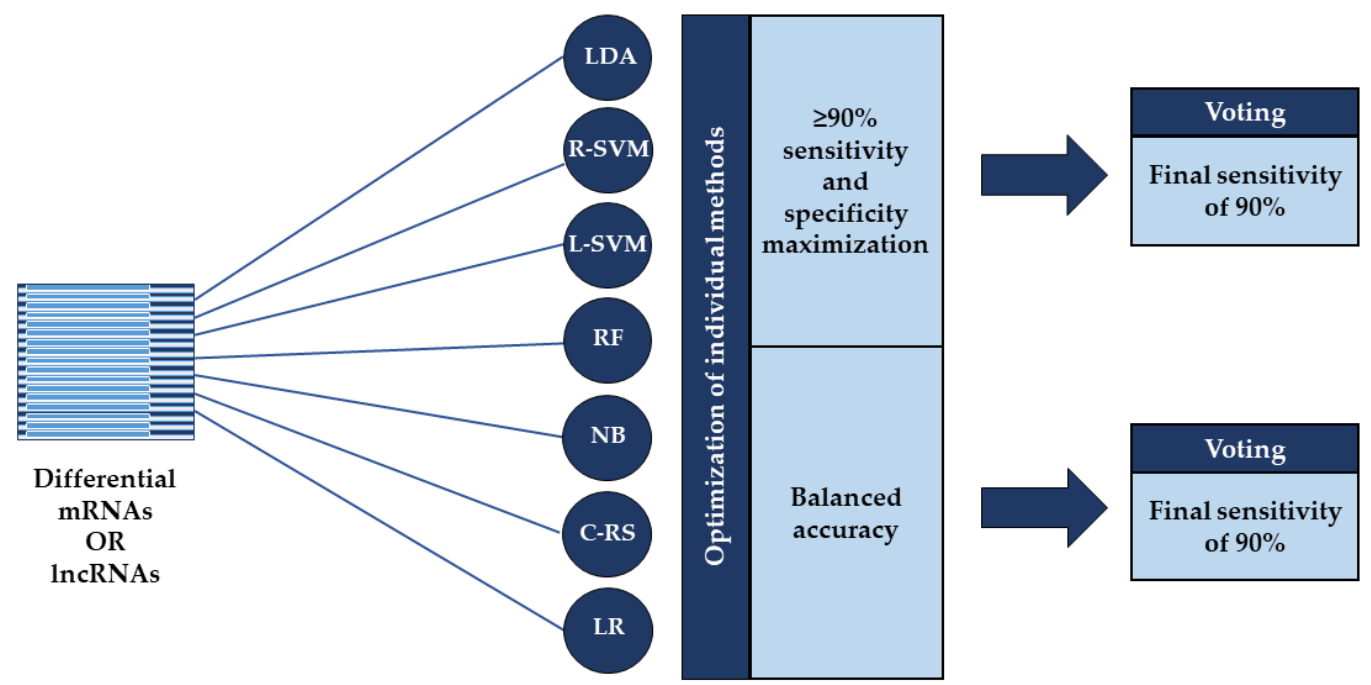

Figure 1. Overview of the two applied classification strategies. Classification was performed according to the differentially expressed mRNAs or lncRNAs using seven machine learning methods: linear discriminant analysis (LDA), support vector machines based on a radial kernel (R-SVM) or linear kernel (L-SVM), random forest (RF), naïve Bayes (NB), COX risk score (C-RS), and logistic regression (LR). The individual methods were optimized using two different approaches: one that provided $\geq 90 \%$ sensitivity while maximizing specificity and one that optimized balanced accuracy where both were followed by voting with a final sensitivity of $90 \%$.

\subsection{Statistical Analysis}

Paired Student's two-tailed $t$-tests were performed for analysis of differential mRNA and lncRNA expression where a false discovery rate (FDR) $\leq 0.05$ was considered significant. Furthermore, a one-sided two-proportion z-test was used to compare the estimated significance between each of the seven methods using lncRNA or mRNA as explanatory variables $\left(\mathrm{H}_{\mathrm{A}}\right.$ : lncRNA performance $>$ mRNA performance) by application of the prop.test function embedded in the stats R-package.

\section{Results}

Gene expression data were analyzed in 160 freshly frozen primary tumors from LNN and systemically untreated low-risk patients with invasive BC. Re-annotation resulted in identification of 21,858 and 4810 mRNA and lncRNA probes on the Agilent array. Further analysis was performed using all mRNA probes and probes covering 2811 unique lncRNAs [26]. Comparing the two patient groups, recurrence development vs. recurrencefree using paired $t$-tests identified 148 and 160 differentially expressed lncRNAs and mRNAs $($ FDR $\leq 0.05)$.

\subsection{Classification with $\geq 90 \%$ Sensitivity Threshold Followed by Voting}

A cumulative risk of recurrence of $\geq 10 \%$ within 10 years is defined as high-risk and in Denmark adjuvant systemic therapy is offered to patients with high risk of recurrence [31]. Classification was therefore conducted with a threshold that provided $\geq 90 \%$ sensitivity to identify patients eligible for systemic treatment. Applying this criterion for the seven methods, classification based on mRNA or lncRNA divided our patients into subgroups with high or low risk of recurrence.

An overall classification accuracy for mRNA data ranged from 53\% (90\% sensitivity, $16 \%$ specificity) using LR to $66 \%$ (91\% sensitivity, 40\% specificity) using RF. For lncRNAbased classification, the overall accuracy ranged from $64 \%$ (91\% sensitivity, 38\% specificity) 
using both the NB and COX-RS methods to 74\% (90\% sensitivity, 58\% specificity) using LDA (Table 2). The specificities obtained by the seven classification methods were consistently higher when using lncRNA compared to mRNA. The classification results with associated $p$-values are shown in Table 2.

Table 2. Classification with $\geq 90 \%$ sensitivity threshold using seven individual machine learning methods.

\begin{tabular}{cccccccc}
\hline \multicolumn{1}{c}{ mRNA } & \multicolumn{5}{c}{ lncRNA } \\
\hline Method & Sensitivity & Specificity & Accuracy $^{\mathbf{a}}$ & Sensitivity & Specificity & Accuracy $^{\mathbf{a}}$ & $p^{\mathbf{b}}$ \\
\hline LDA & 90 & 30 & 60 & 90 & 58 & 74 & 0.0055 \\
\hline R-SVM & 90 & 32 & 61 & 90 & 53 & 71 & 0.038 \\
\hline L-SVM & 91 & 32 & 62 & 90 & 50 & 70 & 0.082 \\
\hline RF & 91 & 40 & 66 & 90 & 52 & 71 & 0.20 \\
\hline NB & 91 & 31 & 61 & 91 & 38 & 64 & 0.33 \\
\hline COX-RS & 90 & 21 & 56 & 91 & 38 & 64 & 0.089 \\
\hline LR & 90 & 16 & 53 & 90 & 50 & 70 & 0.0013 \\
\hline
\end{tabular}

Classification was conducted with a threshold that provided at least $90 \%$ sensitivity while maximizing specificity and performances assessed by leave-one-pair-out cross-validation using linear discriminant analysis (LDA), support vector machines based on a radial kernel (R-SVM) or linear kernel (L-SVM), random forest (RF), naïve Bayes (NB), COX risk score (COX-RS), and logistic regression (LR). ${ }^{a}$ Mean of sensitivity and specificity. All measures are specified in percent. ${ }^{\mathrm{b}} p$-value determined by a one-sided two-proportion $z$-test comparing the estimated significance between the seven machine learning methods using mRNA or lncRNA.

In the clinically most relevant classification scheme, both mRNA and lncRNA-based voting obtained a sensitivity of $91 \%$ at a voting cutoff of 5 where the corresponding specificity was $38 \%$ when mRNA was used and $60 \%$ for lncRNA. The difference between lncRNA and mRNA performance was significant at a $p$-value of 0.013 (Table 3). The individual voting decisions are summarized in Tables S3-S6.

Table 3. Integrated voting results following classification with $\geq 90 \%$ sensitivity threshold.

\begin{tabular}{cccccccc}
\hline \multicolumn{2}{c}{ mRNA } & \multicolumn{5}{c}{ lncRNA } \\
\hline $\begin{array}{c}\text { No. of } \\
\text { Rec. Votes }\end{array}$ & Sensitivity & Specificity & Accuracy $^{\mathbf{a}}$ & Sensitivity & Specificity & Accuracy $^{\mathbf{a}}$ & $p^{\mathbf{b}}$ \\
\hline$\geq 1$ & 100 & 0 & 50 & 98 & 19 & 58 & 0.093 \\
\hline$\geq 2$ & 100 & 10 & 55 & 92 & 29 & 61 & 0.17 \\
\hline$\geq 3$ & 99 & 20 & 59 & 92 & 41 & 67 & 0.086 \\
\hline$\geq 4$ & 96 & 28 & 62 & 92 & 50 & 71 & 0.056 \\
\hline$\geq 5$ & 91 & 38 & 64 & 91 & 60 & 76 & 0.013 \\
\hline$\geq 6$ & 83 & 45 & 64 & 88 & 64 & 76 & 0.013 \\
\hline 7 & 65 & 64 & 64 & 80 & 75 & 78 & 0.0042 \\
\hline
\end{tabular}

The voting results were obtained using seven distinctive cutoffs ranging from 1-7, representing different degrees of classification agreement between the seven machine learning methods in terms of recurrence (rec.) votes. A cutoff of 1 meant that one or more votes for recurrence, placed the patient as high-risk whereas zero votes classified the patient as low-risk and likewise for the rest of the cutoffs. ${ }^{a}$ Mean of sensitivity and specificity. All measures are specified in percent. ${ }^{\mathrm{b}} p$-value determined by a one-sided two-proportion $z$-test comparing the estimated significance between the seven machine learning methods using mRNA or IncRNA. .

\subsection{Classification with Balanced Accuracy Optimization Followed by Voting}

Using optimized bAcc for class assignment by each machine learning method provided an overall classification accuracy for mRNA data ranging from $64 \%(69 \%, 68 \%, 65 \%$ sensitivity; $60 \%, 60 \%, 64 \%$ specificity) using COX-RS, LDA, and LR to $70 \%$ (75\% sensitivity, $65 \%$ specificity) using the NB method. For IncRNA-based classification, the accuracy ranged from $70 \%$ ( $69 \%$ sensitivity, $71 \%$ specificity) using NB to $78 \%$ (79\% sensitivity, $76 \%$ specificity) using the LR method (Table 4). For six of out seven machine learning methods, 
the bAcc were higher when classification was based on lncRNA compared to mRNA, while using NB provided equal performance. The classification results with associated p-values are shown in Table 4.

Table 4. Classification with optimized balanced accuracy using seven individual machine learning methods.

\begin{tabular}{cccccccc}
\hline \multicolumn{7}{c}{ mRNA } & \multicolumn{5}{c}{ lncRNA } \\
\hline Method & Sensitivity & Specificity & Accuracy $^{\mathbf{a}}$ & Sensitivity & Specificity & Accuracy $^{\mathbf{a}}$ & $p^{\mathbf{b}}$ \\
\hline LDA & 68 & 60 & 64 & 75 & 74 & 74 & 0.035 \\
\hline R-SVM & 70 & 66 & 68 & 76 & 68 & 72 & 0.26 \\
\hline L-SVM & 72 & 61 & 67 & 72 & 71 & 72 & 0.20 \\
\hline RF & 71 & 65 & 68 & 75 & 71 & 73 & 0.20 \\
\hline NB & 75 & 65 & 70 & 69 & 71 & 70 & 0.50 \\
\hline COX-RS & 69 & 60 & 64 & 68 & 74 & 71 & 0.11 \\
\hline LR & 65 & 64 & 64 & 79 & 76 & 78 & 0.0042 \\
\hline Cla
\end{tabular}

Classification was conducted with optimized balanced accuracy and performances assessed by leave-one-pair-out cross-validation using linear discriminant analysis (LDA), support vector machines based on a radial kernel (R-SVM) or linear kernel (L-SVM), random forest (RF), naïve Bayes (NB), COX risk score (COX-RS), and logistic regression (LR). ${ }^{a}$ Mean of sensitivity and specificity. All measures are indicated in percent. ${ }^{b} p$-value determined by a one-sided two-proportion z-test comparing the estimated significance between the seven machine learning methods using mRNA or lncRNA.

For the integrated voting results, a sensitivity of $92 \%$ was obtained at a voting cutoff of 1 when mRNA was applied where the corresponding specificity was $29 \%$. At the same cutoff, lncRNA-based voting resulted in a sensitivity of $88 \%$ and specificity of $51 \%$. The voting results with associated p-values are shown in Table 5.

Table 5. Integrated voting results following classification using balanced accuracy optimization.

\begin{tabular}{ccccccccc}
\hline \multicolumn{1}{c}{ mRNA } & \multicolumn{5}{c}{ lncRNA } \\
\hline $\begin{array}{c}\text { No. of } \\
\text { Rec. Votes }\end{array}$ & Sensitivity & Specificity & Accuracy $^{\mathbf{a}}$ & Sensitivity & Specificity & Accuracy $^{\mathbf{a}}$ & $p^{\mathbf{b}}$ \\
\hline $\mathbf{1}$ & 92 & 29 & 61 & 88 & 51 & 69 & 0.083 \\
\hline $\mathbf{2}$ & 82 & 46 & 64 & 84 & 59 & 71 & 0.11 \\
\hline $\mathbf{3}$ & 78 & 62 & 70 & 80 & 65 & 73 & 0.32 \\
\hline $\mathbf{4}$ & 69 & 66 & 68 & 75 & 72 & 74 & 0.14 \\
\hline $\mathbf{2}$ & 62 & 74 & 68 & 70 & 76 & 73 & 0.20 \\
\hline $\mathbf{2}$ & 53 & 85 & 69 & 65 & 88 & 76 & 0.10 \\
\hline $\mathbf{7}$ & 38 & 95 & 66 & 53 & 94 & 73 & 0.11 \\
\hline
\end{tabular}

The voting results were obtained using seven distinctive cutoffs ranging from 1-7, representing different degrees of classification agreement between the seven machine learning methods in terms of recurrence (rec.) votes. A cutoff of 1 meant that one or more votes for recurrence, assigned the patient to the high-risk group whereas zero votes classified the patient as low-risk and similarly for the rest of the cutoffs. ${ }^{a}$ Mean of sensitivity and specificity All measures are indicated in percent. ${ }^{\mathrm{b}} p$-value determined by a one-sided two-proportion $z$-test comparing the estimated significance between the seven machine learning methods using mRNA or lncRNA.

\subsection{Classification of ER Positive Breast Cancer Patients Followed by Voting}

To make our patient group more homogeneous, we considered merely concordant pairs of estrogen receptor (ER) positive patients, retaining a total of 55 patient pairs. Eight hundred and thirty-six mRNAs and 208 lncRNAs were significantly differentially expressed among the ER positive patients (FDR $\leq 0.05$ ). Classification of ER positive BC patients conducted with $\geq 90 \%$ sensitivity, resulted in an overall classification accuracy for mRNA data ranging from 51-68\% whereas the accuracy for lncRNA-based classification ranged from $55-68 \%$ (Table A1). Voting based on mRNA obtained a sensitivity of $96 \%$ at a voting cutoff of 5 where the corresponding specificity was 38\%. At the same cutoff, lncRNA-based 
voting resulted in a sensitivity of $95 \%$ with specificity of $44 \%$. The voting results with associated $p$-values are shown in Table A2.

Classification conducted with optimized bAcc for class assignment resulted in an overall classification accuracy for mRNA data ranging from $62-70 \%$, whereas the accuracy for lncRNA-based classification ranged from $60-69 \%$ (Table A3). Voting using mRNA data obtained a sensitivity of $85 \%$ at a cutoff of 1 where the corresponding specificity was $45 \%$. LncRNA-based voting at the same cutoff resulted in a sensitivity of $91 \%$ with specificity of $36 \%$. The voting results with associated p-values are shown in Table A4.

\section{Discussion}

Our primary objective was to compare the relative ability of mRNA and lncRNA-based signatures to predict recurrence in systemically untreated BC patients using two different classification strategies (Figure 1), which additionally were compared for clinical application. Microarray gene expression analysis identified differentially expressed mRNAs and lncRNAs between matched patient pairs with and without recurrence development where classification subsequently was performed according to these. As the samples were hybridized simultaneously on the same platform, it markedly reduced inter-platform differences when evaluating differential RNA expression and thus strengthened the results.

\subsection{Classification Using IncRNA Compared to mRNA Improved Prognostic Power}

For both mRNA and lncRNA data, and regardless of classification strategy, each of the seven machine learning methods achieved an overall classification accuracy of $>50 \%$, indicating an above random distinction between recurrence developing and recurrence-free patients (Tables 2 and 4). Classification conducted with $\geq 90 \%$ sensitivity, resulted in an overall accuracy for mRNA ranging from $53-66 \%$, whereas the accuracies using lncRNA were noticeably higher, ranging from $64-74 \%$ (Table 2). The same tendency was observed using classification with bAcc optimization, where accuracies for mRNA ranged from $64-70 \%$ and $70-78 \%$ for lncRNA (Table 4). Thus, applying either classification strategy supported the same finding: using the seven machine learning methods, lncRNA compared to mRNA-based signatures improved prognostic accuracy.

For decades, mRNA turnover has been a subject of intensive research and with the characterization of lncRNAs, some similarities and differences between their turnovers have been described. As mature lncRNAs like mRNAs are modified with $5^{\prime}$ caps and $3^{\prime}$-poly adenosine (poly(A)) tails, many mechanisms involved in post-transcriptional mRNA decay are believed to also modulate lncRNA degradation [32]. Other similarities include RNAbinding proteins (RBPs) and microRNAs which have been shown to drive the turnovers of both RNA molecules [33-36]. However, unlike mRNAs, the majority of lncRNAs are not translated into proteins and therefore it is likely that degradation processes associated with the translation machinery are different, leading to individual rates of turnover [32]. Interestingly, some lncRNAs have furthermore been found to form conserved triple-helix complexes which protect them from exonucleases, averting rapid degradation $[37,38]$. The rate of transcription and degradation of mRNAs and lncRNAs ultimately enables their immense impact on gene regulation and their distinct turnover rates might be reflected in our findings of lncRNAs as seemingly better prognostic predictors compared to mRNAs. However, many additional aspects and features of lncRNA turnover still await exploration and characterization by future studies.

\subsection{Classification Followed by Voting Supports $\operatorname{lncRNAs}$ as Better Prognostic Predictors}

To improve classification performance, we applied a voting scheme that allowed the seven methods to vote on whether a sample belonged to the patient group with or without recurrence development. mRNA-based accuracies derived from the integrated voting results following classification with $\geq 90 \%$ sensitivity threshold, ranged from $50-64 \%$, whereas higher accuracies of 58-78\% were obtained for lncRNA (Table 3). The integrated voting results after classification using the alternative strategy of bAcc optimization also 
supported lncRNAs as better prognostic predictors with accuracies ranging from 69-76\% for lncRNA while mRNA-based voting obtained 61-70\% (Table 5).

The number of studies comparing the relative ability of mRNA and lncRNA-based signatures to predict recurrence are very limited. However, a study by $\mathrm{Xu}$ et al., aimed to identify biomarkers that could improve prognostic predictions [39]. By comparing the expression of IncRNA, mRNA, microRNA, and DNA methylation, they found IncRNAs to be the best prognostic predictors in validated cohorts of four cancer types including BC [39], supporting our findings. However, of the breast tumors included in their study (with comprehensive clinicopathological information), more than half were lymph node positive. Furthermore, they had a short median overall follow-up of 17 months and a small fraction of overall survival events ( 93 out of 818 patients). To the best of our knowledge, no other studies have performed these comparisons in LNN and systemically untreated BC patients with very long follow-up.

\subsection{Comparison of the Two Voting Strategies for Clinical Signature Development}

If we could improve prediction of recurrence at time of diagnosis, a more accurate prognosis could be established for low-risk BC patients and we would thereby achieve substantial reduction of adjuvant systemic therapy application [12-15]. To elucidate and compare clinical application of the two classification strategies for future signature development, we considered the amount of correctly classified patients in the two groups. We would consistently spare more recurrence-free patients unnecessary adjuvant systemic therapy using lncRNA compared to mRNA-based voting following classification with $\geq 90 \%$ sensitivity. Applying this threshold for lncRNA-based voting with a cutoff of 5, we correctly spare 48 out of $80(60 \%)$ recurrence-free patients adjuvant systemic therapy while correctly classifying 73 out of $80(91 \%)$ patients with recurrence development. Compared to mRNA-based voting, it is 18 additional patients that possibly could have been taken off treatment today ( $60 \%$ vs. $38 \%$ specificity) while maintaining a similar sensitivity of $91 \%$ ( $p$-value of 0.0013 , Table 3).

Making the strategy of optimized bAcc for lncRNA-based voting equally relevant in terms of clinical application, thus, applying a cutoff of 1 , we correctly spare 41 out of $80(51 \%)$ recurrence-free patients unnecessary therapy while correctly classifying 70 out of $80(88 \%)$ recurrence developing patients. Compared to mRNA-based voting, it is similarly 18 additional patients which possibly could have been taken off treatment today ( $51 \%$ vs. $29 \%$ specificity), although at a slight cost of sensitivity ( $88 \%$ vs. $92 \%$ sensitivity, $p$-value of 0.083 , Table 5).

In a clinical situation, the goal would be a final sensitivity after voting of close to $90 \%$, i.e., nearly all patients who develop metastases need to be classified as high-risk. For which of the two initial classification schemes in the individual machine learning methods: (1) Optimization of specificity with sensitivity set at 90\% (Table 2); or (2) Optimization of bAcc (Table 4), can we obtain the highest final specificity (correct classification of patients as low-risk)? By comparing results shown in Tables 3 and 5, it can be seen that highest specificity (with final sensitivity fixed at $90 \%$ ) can be obtained when the sensitivity was fixed at $90 \%$ in the seven initial classifiers.

\subsection{Comparison of the Two Voting Strategies for Clinical Application in ER Positive Patients}

For ER positive BC patients, classification followed by voting conducted with $\geq 90 \%$ sensitivity could likewise spare more recurrence-free patients unnecessary adjuvant systemic therapy using IncRNA compared to mRNA while achieving almost similar sensitivity (Table A2). As our patient group became more homogeneous and as the classification models were developed in a majority of ER positive samples, we could expect this finding. The increased sample homogeneity was also reflected in identification of a larger amount of differentially expressed RNA molecules in ER positive patients.

The potential advantages of IncRNAs compared to mRNAs was however not as distinctive when applying the alternative strategy of bAcc optimization, indicating that the 
fewer ER negative samples contributed to a more heterogeneous data structure, leading to better distinction between the two patient groups when using lncRNA data compared to mRNA. LncRNA-based voting was still favored for clinical application when using this strategy as it, unlike mRNA, enabled a sensitivity of $\geq 90 \%$ (Table A4).

\subsection{LncRNAs and Their Countless Roles}

Although we have more than seven times as many unique mRNA than IncRNA probes on the array (21,858 vs. 2811), paired Student's two-tailed $t$-tests identified almost the same number of differentially expressed mRNAs and lncRNAs (160 and 148) and thus, a much higher percentage of lncRNAs were found to be statistically significant. Considering this, along with the mentioned findings of lncRNAs as seemingly better prognostic predictors of recurrence in low-risk BC patients, lncRNAs are suggested to be far more informative than mRNAs, indicating that they must have crucial roles in BC progression.

Several links between lncRNAs and cancer are now known where alteration of lncRNA expression in cancer cells have been demonstrated in multiple cancers besides BC, e.g., prostate and non-small cell lung cancer [23,40-43]. It is however very difficult to generalize findings across different cancer types in different clinical settings.

The roles of lncRNAs are vast and intricate as they interact with DNA elements, mRNAs, microRNAs, and proteins where their multifunctional regulatory roles are exerted on multiple levels: epigenetic, peptide-mediated, transcriptional, and post-transcriptional [44]. The diversity of their regulatory roles also includes crucial functions throughout the steps of the metastatic cascade. Different lncRNAs-e.g., H19 and PTAR-have been described in the step of epithelial-mesenchymal transition whereas others-e.g., NORAD and MALAT1 - are involved in the promotion of invasion and colonization, respectively (as reviewed by Liu et al. [45]). Exciting lncRNA discoveries hold for future studies where a profound comprehension of the functional roles and mechanisms of lncRNAs along with their complex regulatory network will be explored.

\section{Conclusions}

The majority of gene expression signatures providing prognostic information in BC have primarily been developed on mRNAs with a few on lncRNAs. The lack of studies comparing such signatures in low-risk BC patients make this study highly relevant.

In the clinically most relevant classification scheme both mRNA and lncRNA-based voting obtained a sensitivity of $91 \%$ at a voting cutoff of 5 where the corresponding specificity was 38\% when mRNA was used and $60 \%$ for lncRNA. The difference between lncRNA and mRNA performance was significant at a $p$-value of 0.013 (Table 3 ).

Classification with a fixed sensitivity of $\geq 90 \%$ for the individual machine learning methods followed by voting with a final sensitivity of $90 \%$, obtained consistently higher overall accuracies when based on lncRNAs compared to mRNAs. Similar findings were observed using the alternative strategy of bAcc optimization and thus, classification followed by voting suggested improved prognostic power using lncRNAs compared to mRNAs. Comparing the two classification strategies for clinical application, suggested that development of future RNA-based signatures for assisting clinical decision-making, could gain prognostic power using lncRNA-based classification with $\geq 90 \%$ sensitivity followed by voting with a final sensitivity of $90 \%$.

In summary, our data suggest that in a group of BC patients, lncRNAs are more informative than mRNAs in prediction of recurrence. We additionally propose a favorable optimizing and classification strategy and thus, we hope that these data encourage other research groups to at least include lncRNAs for their signature development in low-risk BC.

Supplementary Materials: The following are available online at https:/ / www.mdpi.com/article/10 .3390/ cancers13194907/s1, Table S1: Significant mRNAs. Table S2: Annotated significant lncRNAs. Table S3: mRNA-based classification decisions. Table S4: LncRNA-based classification decisions. Table S5: Classification decisions based on mRNAs and lncRNAs, sorted by lncRNAs. Table S6: Classification decisions based on mRNAs and lncRNAs, sorted by mRNAs. 
Author Contributions: Conceptualization, T.A.K. and M.T.; Methodology, T.A.K., M.T., Q.T. and M.B. (Mark Burton); Software, M.B. (Mark Burton); Formal analysis, M.B. (Mark Burton); Resources, M.B. (Martin Bak), S.C.; Data curation, M.B. (Mark Burton), M.J.L., I.B. and K.P.S.; Writ-ing—original draft preparation, T.T.N.D. and I.B.; Writing-review and editing, T.T.N.D., M.B. (Mark Burton), I.B., M.T. and T.A.K.; Visualization, T.T.N.D. and I.B.; Supervision, T.A.K. and M.T.; Project administration, T.A.K.; Funding acquisition, T.A.K., M.T. and K.P.S. All authors have read and agreed to the published version of the manuscript.

Funding: This research was funded by the Danish Cancer Society, the Danish Council for Independent Research (grants: 7016-00346B/FSS and 09-061677/FSS), the Danish Ministry of the Interior, the University of Southern Denmark (Horizon2020, grant: DAWN2020), the Danish Council for Strategic Research (grant: DBCG-TIBCAT) and Regionernes Medicinpulje, Dansk Kræftforskningsfond, Breast Friends, Fonden til Lægevidenskabens Fremme, Meta \& Håkon Baggers Fond, A. J. Andersen \& hustrus Fond, Inge \& Jørgen Larsens Mindelegat, Overlægerådets Legatudvalg, Direktør Jacob Madsens \& Hustru Olga Madsens Fond, Fru Ingeborg Anna Albinus Larsens Mindelegat, Fonden af 1870, Harboefonden, the Odense University Hospital Fund for Free Research, Lundbeckfonden (grant: Center of Excellence NanoCAN), and Frimodt-Heineke Fonden.

Institutional Review Board Statement: The study was approved by the Danish National Committee on Health Research (S-VF-20020142).

Informed Consent Statement: Patient consent was waived because the tumor samples included in the study were collected from established biobanks at Odense University Hospital, Denmark where utilization of these surplus samples has been approved by the Danish National Committee on Health Research Ethics. Patients who objected to the use of their tumor material via the Danish Tissue Utilization Register were excluded from the study.

Data Availability Statement: The data presented in this study are openly available in the National Center of Biotechnology Information's Gene Expression Omnibus [24] and are accessible through GEO Series accession number GSE48408 [19].

Conflicts of Interest: The authors declare no conflict of interest. The funders had no role in the design of the study; in the collection, analyses, or interpretation of data; in the writing of the manuscript, or in the decision to publish the results.

\section{Appendix A}

Appendix Methods

Classification

We performed classification according to the differentially expressed RNA molecules using seven machine learning methods and applying two different approaches in which the first provided $\geq 90 \%$ sensitivity while maximizing specificity whereas the other optimized balanced accuracy. Both strategies were followed by voting with a final sensitivity of $90 \%$. For the first approach, the optimal model during training was selected based on a fixed sensitivity of $\geq 90 \%$ while the specificity was maximized, assessed by leave-onepair-out cross-validation (LOPOCV). Using the second approach, the optimal model was determined based on mean sensitivity and specificity maximization, likewise assessed by LOPOCV.

In brief, LOPOCV is a procedure that evaluates how well a machine learning method performs by using a single pair of matched samples as test samples, while the remaining samples serve as a training set. Each single pair of matched samples was thus tested individually, and the procedure was repeated until all pairs of matched samples had been left out once. The accuracy of the machine learning methods was determined by the amount of correctly classified samples. An unbiased performance estimate is provided by this procedure, and this approach has furthermore proven to be optimal for analysis of smaller datasets $[27,28]$.

Feature selection is required in the training set to avoid a small sample-per-feature ratio and it has been shown to provide better classification [28]. In this study, the feature selection process comprised of three steps: 1) Testing the mRNAs and lncRNAs in the 
training set for significant differential expression using paired Student's two-tailed $t$-test (FDR $\leq 0.05) ; 2)$ Ranking the differentially expressed mRNAs and lncRNAs according to their random-forest importance value which describes the standardized drop in prediction accuracy when the class levels are permuted [46];3) By subsequently adding one mRNA or lncRNA at a time in a top-down forward-wrapper approach starting with top two mRNAs or lncRNAs from the ranked list followed by classification accuracy assessment. The optimal number of mRNAs and lncRNAs were determined by each of the seven machine learning methods. At each addition, the classification accuracy of the training samples was evaluated using LOPOCV in a nested inner loop [29]. To calculate the significance of the classification results, a one-sided two-proportion z-test was applied, comparing the estimated significance between the seven machine learning methods using mRNA or lncRNA. The pipeline for these classification steps is shown in Figure A1.

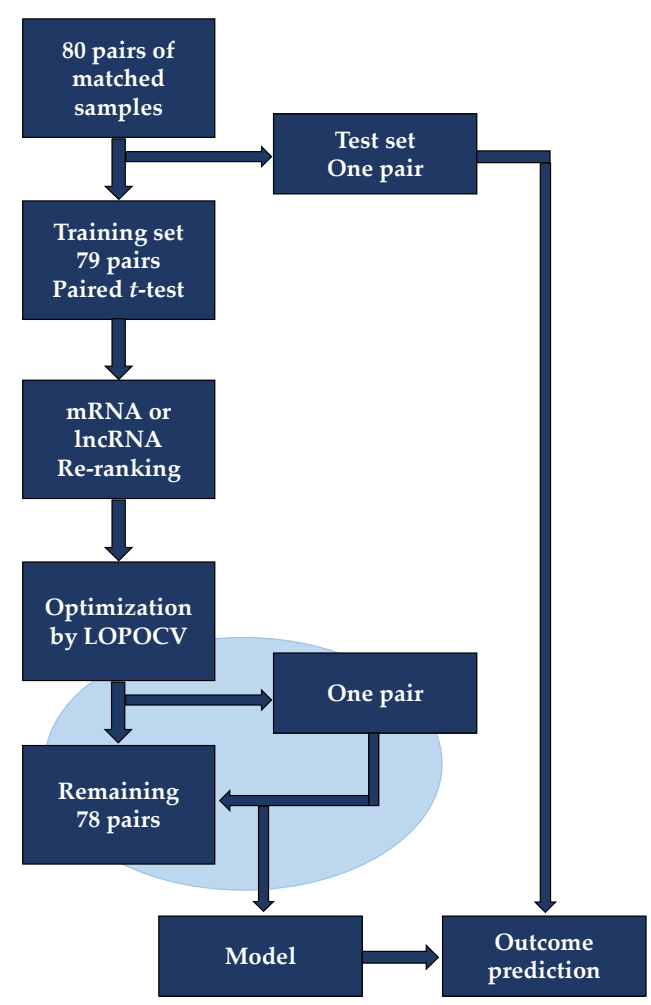

Figure A1. Outcome prediction using leave-one-pair-out cross-validation. In this procedure, a single pair of matched samples served as test samples while the remaining 79 sample pairs served as a training set. For feature selection in the training set, Student's two-tailed $t$-test was used to identify differentially expressed mRNAs and lncRNAs (FDR $\leq 0.05)$ where these subsequently were re-ranked according to their random forest-based variable importance value. Using leave-one-outcross-validation (LOPOCV), each of the seven machine learning methods identified the optimal mRNA and lncRNA set by using the highest ranked mRNAs/ lncRNAs in a top-down forwardwrapper approach where outcome in one selected pair was predicted using the remaining 78 pairs as training set (inner loop, blue). The inner loop procedure was repeated until each pair had been left out once. The final optimal mRNA or lncRNA set was used to predict outcome in the initially left out test pair. The entire procedure was repeated until all pairs had been left out as a test pair and their outcome independently predicted by the inner training loop. 
Table A1. Classification of ER positive BC patients with $\geq 90 \%$ sensitivity threshold using seven individual machine learning methods.

\begin{tabular}{cccccccc}
\hline \multicolumn{9}{c}{ mRNA } & \multicolumn{5}{c}{ lncRNA } \\
\hline Method & Sensitivity & Specificity & Accuracy $^{\mathbf{a}}$ & Sensitivity & Specificity & Accuracy $^{\mathbf{a}}$ & $\boldsymbol{p}^{\mathbf{b}}$ \\
\hline LDA & 98 & 16 & 57 & 95 & 31 & 63 & 0.22 \\
\hline R-SVM & 96 & 40 & 68 & 91 & 35 & 63 & 0.74 \\
\hline L-SVM & 91 & 42 & 66 & 91 & 38 & 65 & 0.51 \\
\hline RF & 91 & 31 & 61 & 93 & 44 & 68 & 0.17 \\
\hline NB & 91 & 35 & 63 & 93 & 36 & 65 & 0.43 \\
\hline COX-RS & 91 & 11 & 51 & 91 & 35 & 63 & 0.048 \\
\hline LR & 91 & 15 & 53 & 93 & 16 & 55 & 0.44
\end{tabular}

Classification was conducted with a threshold that provided at least $90 \%$ sensitivity while maximizing specificity and performances assessed by leave-one-pair-out cross-validation using linear discriminant analysis (LDA), support vector machines based on a radial kernel (R-SVM) or linear kernel (L-SVM), random forest (RF), naïve Bayes (NB), COX risk score (COX-RS), and logistic regression (LR). ${ }^{\text {a }}$ Mean of sensitivity and specificity. All measures are indicated in percent. ${ }^{\mathrm{b}} p$-value determined by a one-sided two-proportion z-test comparing the estimated significance between the seven machine learning methods using mRNA or lncRNA.

Table A2. Integrated voting results for ER positive BC patients following classification with $\geq 90 \%$ sensitivity threshold.

\begin{tabular}{cccccccc}
\hline \multicolumn{1}{c}{ mRNA } & \multicolumn{5}{c}{ lncRNA } \\
\hline $\begin{array}{c}\text { No. of } \\
\text { Rec. Votes }\end{array}$ & Sensitivity & Specificity & Accuracy $^{\mathbf{a}}$ & Sensitivity & Specificity & Accuracy $^{\mathbf{a}}$ & $p^{\mathbf{b}}$ \\
\hline$\geq 1$ & 100 & 0 & 50 & 100 & 2 & 51 & 0.49 \\
\hline$\geq 2$ & 100 & 2 & 51 & 100 & 9 & 55 & 0.32 \\
\hline$\geq 3$ & 98 & 13 & 55 & 98 & 18 & 58 & 0.38 \\
\hline$\geq 4$ & 98 & 18 & 58 & 98 & 33 & 65 & 0.18 \\
\hline$\geq 5$ & 96 & 38 & 67 & 95 & 44 & 69 & 0.43 \\
\hline$\geq 6$ & 87 & 49 & 68 & 87 & 60 & 74 & 0.20 \\
\hline 7 & 69 & 69 & 69 & 71 & 73 & 72 & 0.37
\end{tabular}

Voting was conducted using seven distinctive cutoffs ranging from 1-7, representing different degrees of classification agreement between the seven machine learning methods in terms of recurrence (rec.) votes. A cutoff of 1 meant that one or more votes for recurrence, allocated the patient to the high-risk group whereas zero votes classified the patient as low-risk and likewise for the rest of the cutoffs. ${ }^{a}$ Mean of sensitivity and specificity. All measures are indicated in percent. ${ }^{\mathrm{b}} p$-value determined by a one-sided two-proportion z-test comparing the estimated significance between the seven machine learning methods using mRNA or lncRNA.

Table A3. Classification of ER positive BC patients with optimized balanced accuracy using seven individual classifiers.

\begin{tabular}{cccccccc}
\hline \multicolumn{7}{c}{ mRNA } & \multicolumn{5}{c}{ lncRNA } \\
\hline Method & Sensitivity & Specificity & Accuracy $^{\mathbf{a}}$ & Sensitivity & Specificity & Accuracy $^{\mathbf{a}}$ & $p^{\mathbf{b}}$ \\
\hline LDA & 62 & 62 & 62 & 56 & 65 & 61 & 0.51 \\
\hline R-SVM & 75 & 65 & 70 & 69 & 69 & 69 & 0.51 \\
\hline L-SVM & 67 & 71 & 69 & 60 & 73 & 66 & 0.63 \\
\hline RF & 67 & 64 & 65 & 65 & 67 & 66 & 0.49 \\
\hline NB & 62 & 76 & 69 & 62 & 71 & 66 & 0.63 \\
\hline COX-RS & 62 & 76 & 69 & 58 & 62 & 60 & 0.90 \\
\hline LR & 60 & 75 & 67 & 56 & 75 & 65 & 0.56 \\
\hline
\end{tabular}

Classification was conducted with optimized balanced accuracy and performances assessed by leave-one-pair-out cross-validation using linear discriminant analysis (LDA), support vector machines based on a radial kernel (R-SVM) or linear kernel (L-SVM), random forest (RF), naïve Bayes (NB), COX risk score (COX-RS), and logistic regression (LR). ${ }^{\text {a }}$ Mean of sensitivity and specificity. All measures are specified in percent. ${ }^{b} p$-value determined by a one-sided two-proportion z-test comparing the estimated significance between the seven machine learning methods using mRNA or lncRNA. 
Table A4. Integrated voting results for ER positive BC patients following optimized balanced accuracy classification.

\begin{tabular}{|c|c|c|c|c|c|c|c|}
\hline \multicolumn{4}{|c|}{ mRNA } & \multicolumn{4}{|c|}{ lncRNA } \\
\hline $\begin{array}{c}\text { No. of } \\
\text { Rec. Votes }\end{array}$ & Sensitivity & Specificity & Accuracy $^{a}$ & Sensitivity & Specificity & Accuracy ${ }^{a}$ & $p^{\mathrm{b}}$ \\
\hline$\geq 1$ & 85 & 45 & 65 & 91 & 36 & 64 & 0.51 \\
\hline$\geq 2$ & 84 & 53 & 68 & 85 & 56 & 71 & 0.37 \\
\hline$\geq 3$ & 75 & 67 & 71 & 76 & 62 & 69 & 0.57 \\
\hline$\geq 4$ & 65 & 71 & 68 & 64 & 73 & 68 & 0.50 \\
\hline$\geq 5$ & 60 & 78 & 69 & 53 & 80 & 66 & 0.63 \\
\hline$\geq 6$ & 56 & 85 & 71 & 36 & 85 & 61 & 0.92 \\
\hline 7 & 27 & 95 & 61 & 22 & 89 & 55 & 0.78 \\
\hline
\end{tabular}

The voting results were obtained using seven distinctive cutoffs ranging from 1-7, representing different degrees of classification agreement between the seven machine learning methods in terms of recurrence (rec.) votes. A cutoff of 1 meant that one or more votes for recurrence, placed the patient as high-risk whereas zero votes classified the patient as low-risk and similarly for the rest of the cutoffs. a Mean of sensitivity and specificity. All measures are indicated in percent. ${ }^{\mathrm{b}} p$-value determined by a one-sided two-proportion $\mathrm{z}$-test comparing the estimated significance between the seven machine learning methods using mRNA or lncRNA.

\section{References}

1. Sørlie, T.; Perou, C.M.; Tibshirani, R.; Aas, T.; Geisler, S.; Johnsen, H.; Hastie, T.; Eisen, M.B.; van de Rijn, M.; Jeffrey, S.S.; et al. Gene expression patterns of breast carcinomas distinguish tumor subclasses with clinical implications. Proc. Natl. Acad. Sci. USA 2001, 98, 10869-10874. [CrossRef]

2. Elston, C.W.; Ellis, I.O. Pathological prognostic factors in breast cancer. I. The value of histological grade in breast cancer: Experience from a large study with long-term follow-up. Histopathology 1991, 19, 403-410. [CrossRef]

3. Giuliano, A.E.; Connolly, J.L.; Edge, S.B.; Mittendorf, E.A.; Rugo, H.S.; Solin, L.J.; Weaver, D.L.; Winchester, D.J.; Hortobagyi, G.N. Breast Cancer-Major changes in the American Joint Committee on Cancer eighth edition cancer staging manual. CA Cancer J. Clin. 2017, 67, 290-303. [CrossRef]

4. Dexter, D.L.; Kowalski, H.M.; Blazar, B.A.; Fligiel, Z.; Vogel, R.; Heppner, G.H. Heterogeneity of tumor cells from a single mouse mammary tumor. Cancer Res. 1978, 38, 3174-3181.

5. Shah, S.P.; Morin, R.D.; Khattra, J.; Prentice, L.; Pugh, T.; Burleigh, A.; Delaney, A.; Gelmon, K.; Guliany, R.; Senz, J.; et al. Mutational evolution in a lobular breast tumour profiled at single nucleotide resolution. Nature 2009, 461, 809-813. [CrossRef]

6. Cajal, S.R.Y.; De Mattos-Arruda, L.; Sonenberg, N.; Cortes, J.; Peg, V. The intra-tumor heterogeneity of cell signaling factors in breast cancer: p4E-BP1 and peIF4E are diffusely expressed and are real potential targets. Clin. Transl. Oncol. 2014, 16, 937-941. [CrossRef]

7. Fidler, I.J.; Kripke, M.L. Metastasis results from preexisting variant cells within a malignant tumor. Science 1977, 197, 893-895. [CrossRef] [PubMed]

8. Fidler, I.J. Tumor heterogeneity and the biology of cancer invasion and metastasis. Cancer Res. 1978, 38, 2651-2660. [CrossRef]

9. Cardoso, F.; Kyriakides, S.; Ohno, S.; Penault-Llorca, F.; Poortmans, P.; Rubio, I.T.; Zackrisson, S.; Senkus, E. Early breast cancer: ESMO Clinical Practice Guidelines for diagnosis, treatment and follow-up. Ann. Oncol. 2019, 30, 1194-1220. [CrossRef] [PubMed]

10. Mouridsen, H.T.; Bjerre, K.D.; Christiansen, P.; Jensen, M.-B.; Møller, S. Improvement of prognosis in breast cancer in Denmark 1977-2006, based on the nationwide reporting to the DBCG Registry. Acta Oncol. 2008, 47, 525-536. [CrossRef] [PubMed]

11. Synnestvedt, M.; Borgen, E.; Russnes, H.G.; Kumar, N.T.; Schlichting, E.; Giercksky, K.-E.; Kåresen, R.; Nesland, J.M.; Naume, B. Combined analysis of vascular invasion, grade, HER2 and Ki67 expression identifies early breast cancer patients with questionable benefit of systemic adjuvant therapy. Acta Oncol. 2013, 52, 91-101. [CrossRef] [PubMed]

12. Offersen, B.V.; Ejlertsen, B.; Balslev, E.; Flyger, H.; Gerdes, A.-M.; Hansen, M.K.; Hølmich, L.R.; Jensen, M.-B.; Kristensen, B.; Laenkholm, A.-V.; et al. Is DBCG abreast of new developments? Acta Oncol. 2018, 57, 1-2. [CrossRef] [PubMed]

13. Delahaye, L.J.M.J.; Drukker, C.A.; Dreezen, C.; Witteveen, A.; Chan, B.; Snel, M.; Beumer, I.J.; Bernards, R.; Audeh, M.W.; Van't Veer, L.J.; et al. A breast cancer gene signature for indolent disease. Breast Cancer Res. Treat. 2017, 164, 461-466. [CrossRef] [PubMed]

14. Esserman, L.J.; Yau, C.; Thompson, C.K.; van 't Veer, L.J.; Borowsky, A.D.; Hoadley, K.A.; Tobin, N.P.; Nordenskjöld, B.; Fornander, T.; Stål, O.; et al. Use of Molecular Tools to Identify Patients With Indolent Breast Cancers With Ultralow Risk Over 2 Decades. JAMA Oncol. 2017, 3, 1503-1510. [CrossRef] [PubMed]

15. Sjöström, M.; Chang, S.L.; Fishbane, N.; Davicioni, E.; Hartman, L.; Holmberg, E.; Feng, F.Y.; Speers, C.; Pierce, L.J.; Malmström, P.; et al. Comprehensive transcriptomic profiling identifies breast cancer patients who may be spared adjuvant systemic therapy. Clin. Cancer Res. 2019, 26, 171-182. [CrossRef] [PubMed] 
16. Van 't Veer, L.J.; Dai, H.; Van de Vijver, M.J.; He, Y.D.; Hart, A.A.M.; Mao, M.; Peterse, H.L.; van der Kooy, K.; Marton, M.J.; Witteveen, A.T.; et al. Gene expression profiling predicts clinical outcome of breast cancer. Nature 2002, 415, 530-536. [CrossRef]

17. Sparano, J.A.; Gray, R.J.; Makower, D.F.; Pritchard, K.I.; Albain, K.S.; Hayes, D.F.; Geyer, C.E.; Dees, E.C.; Goetz, M.P.; Olson, J.A.; et al. Adjuvant Chemotherapy Guided by a 21-Gene Expression Assay in Breast Cancer. N. Engl. J. Med. 2018, 379, 111-121. [CrossRef]

18. Parker, J.S.; Mullins, M.; Cheang, M.C.U.; Leung, S.; Voduc, D.; Vickery, T.; Davies, S.; Fauron, C.; He, X.; Hu, Z.; et al. Supervised risk predictor of breast cancer based on intrinsic subtypes. J. Clin. Oncol. 2009, 27, 1160-1167. [CrossRef]

19. Sørensen, K.; Thomassen, M.; Tan, Q.; Bak, M.; Cold, S.; Burton, M.; Larsen, M.; Kruse, T. Long non-coding RNA expression profiles predict metastasis in lymph node-negative breast cancer independently of traditional prognostic markers. Breast Cancer Res. 2015, 17, 55. [CrossRef]

20. Li, J.; Wang, W.; Xia, P.; Wan, L.; Zhang, L.; Yu, L.; Wang, L.; Chen, X.; Xiao, Y.; Xu, C. Identification of a five-lncRNA signature for predicting the risk of tumor recurrence in patients with breast cancer. Int. J. Cancer 2018, 143, 2150-2160. [CrossRef]

21. Zhang, Y.; Li, Z.; Chen, M.; Chen, H.; Zhong, Q.; Liang, L.; Li, B. Identification of a New Eight-Long Noncoding RNA Molecular Signature for Breast Cancer Survival Prediction. DNA Cell Biol. 2019, 38, 1529-1539. [CrossRef] [PubMed]

22. Borkiewicz, L.; Kalafut, J.; Dudziak, K.; Przybyszewska-Podstawka, A.; Telejko, I. Decoding LncRNAs. Cancers 2021, 13, 2643. [CrossRef] [PubMed]

23. Sørensen, K.P.; Thomassen, M.; Tan, Q.; Bak, M.; Cold, S.; Burton, M.; Larsen, M.J.; Kruse, T.A. Long non-coding RNA HOTAIR is an independent prognostic marker of metastasis in estrogen receptor-positive primary breast cancer. Breast Cancer Res. Treat. 2013, 142, 529-536. [CrossRef] [PubMed]

24. Edgar, R.; Domrachev, M.; Lash, A.E. Gene Expression Omnibus: NCBI gene expression and hybridization array data repository. Nucleic Acids Res. 2002, 30, 207-210. [CrossRef] [PubMed]

25. GENCODE. Available online: https:/ / www.gencodegenes.org/ (accessed on 6 August 2013).

26. Tørring, P.M.; Larsen, M.J.; Kjeldsen, A.D.; Ousager, L.B.; Tan, Q.; Brusgaard, K. Long non-coding RNA expression profiles in hereditary haemorrhagic telangiectasia. PLoS ONE 2014, 9, e90272. [CrossRef] [PubMed]

27. Cruz, J.A.; Wishart, D.S. Applications of machine learning in cancer prediction and prognosis. Cancer Inform. 2007, 2, 59-77. [CrossRef]

28. Somorjai, R.L.; Dolenko, B.; Baumgartner, R. Class prediction and discovery using gene microarray and proteomics mass spectroscopy data: Curses, caveats, cautions. Bioinformatics 2003, 19, 1484-1491. [CrossRef]

29. Burton, M.; Thomassen, M.; Tan, Q.; Kruse, T.A. Gene expression profiles for predicting metastasis in breast cancer: A cross-study comparison of classification methods. Sci. World J. 2012, 2012, 380495. [CrossRef]

30. Newcombe, R.G. Simultaneous comparison of sensitivity and specificity of two tests in the paired design: A straightforward graphical approach. Stat. Med. 2001, 20, 907-915. [CrossRef]

31. DBCG, Danish Breast Cancer Cooperative Group. Medical treatment-Selection for systemical treatment. Summary of DBCG's recommendations (in Danish) [Internet]. 2019. Available online: https://dbcg.dk/images/PDF/Retningslinier/Kap_6_Medicinsk_ behandling-21.02.2019.pdf (accessed on 28 September 2021).

32. Yoon, J.-H.; Kim, J.; Gorospe, M. Long noncoding RNA turnover. Biochimie 2015, 117, 15-21. [CrossRef]

33. Yoon, J.-H.; De, S.; Srikantan, S.; Abdelmohsen, K.; Grammatikakis, I.; Kim, J.; Kim, K.M.; Noh, J.H.; White, E.J.F.; Martindale, J.L.; et al. PAR-CLIP analysis uncovers AUF1 impact on target RNA fate and genome integrity. Nat. Commun. 2014, 5, 5248. [CrossRef]

34. Kang, M.-J.; Abdelmohsen, K.; Hutchison, E.R.; Mitchell, S.J.; Grammatikakis, I.; Guo, R.; Noh, J.H.; Martindale, J.L.; Yang, X.; Lee, E.K.; et al. HuD Regulates Coding and Noncoding RNA to Induce APP $\rightarrow$ A $\beta$ Processing. Cell Rep. 2014, 7, 1401-1409. [CrossRef] [PubMed]

35. Bagga, S.; Bracht, J.; Hunter, S.; Massirer, K.; Holtz, J.; Eachus, R.; Pasquinelli, A.E. Regulation by let-7 and lin-4 miRNAs Results in Target mRNA Degradation. Cell 2005, 122, 553-563. [CrossRef] [PubMed]

36. Leucci, E.; Patella, F.; Waage, J.; Holmstrøm, K.; Lindow, M.; Porse, B.; Kauppinen, S.; Lund, A.H. microRNA-9 targets the long non-coding RNA MALAT1 for degradation in the nucleus. Sci. Rep. 2013, 3, 2535. [CrossRef] [PubMed]

37. Wilusz, J.E.; JnBaptiste, C.K.; Lu, L.Y.; Kuhn, C.-D.; Joshua-Tor, L.; Sharp, P.A. A triple helix stabilizes the $3^{\prime}$ ends of long noncoding RNAs that lack poly(A) tails. Genes Dev. 2012, 26, 2392-2407. [CrossRef] [PubMed]

38. Brown, J.A.; Valenstein, M.L.; Yario, T.A.; Tycowski, K.T.; Steitz, J.A. Formation of triple-helical structures by the $3^{\prime}$-end sequences of MALAT1 and MEN $\beta$ noncoding RNAs. Proc. Natl. Acad. Sci. USA 2012, 109, 19202-19207. [CrossRef]

39. Xu, L.; Fengii, L.; Changning, L.; Li, X.; Yinghui, L.; Yu, L.; Shanguang, C.; Jianghui, X. Comparison of the Prognostic Utility of the Diverse Molecular Data among lncRNA, DNA Methylation, microRNA, and mRNA across Five Human Cancers. PLoS ONE 2015, 10, e0142433. [CrossRef]

40. Gupta, R.A.; Shah, N.; Wang, K.C.; Kim, J.; Horlings, H.M.; Wong, D.J.; Tsai, M.-C.; Hung, T.; Argani, P.; Rinn, J.L.; et al. Long non-coding RNA HOTAIR reprograms chromatin state to promote cancer metastasis. Nature 2010, 464, 1071-1076. [CrossRef]

41. Bussemakers, M.J.; van Bokhoven, A.; Verhaegh, G.W.; Smit, F.P.; Karthaus, H.F.; Schalken, J.A.; Debruyne, F.M.; Ru, N.; Isaacs, W.B. DD3: A new prostate-specific gene, highly overexpressed in prostate cancer. Cancer Res. 1999, 59, 5975-5979.

42. Ji, P.; Diederichs, S.; Wang, W.; Böing, S.; Metzger, R.; Schneider, P.M.; Tidow, N.; Brandt, B.; Buerger, H.; Bulk, E.; et al. MALAT-1, a novel noncoding RNA, and thymosin beta4 predict metastasis and survival in early-stage non-small cell lung cancer. Oncogene 2003, 22, 8031-8041. [CrossRef] 
43. Prensner, J.R.; Iyer, M.K.; Sahu, A.; Asangani, I.A.; Cao, Q.; Patel, L.; Vergara, I.A.; Davicioni, E.; Erho, N.; Ghadessi, M.; et al. The long noncoding RNA SChLAP1 promotes aggressive prostate cancer and antagonizes the SWI/SNF complex. Nat. Genet. 2013, 45, 1392-1398. [CrossRef] [PubMed]

44. Chen, X.; Sun, Y.; Cai, R.; Wang, G.; Shu, X.; Pang, W. Long noncoding RNA: Multiple players in gene expression. BMB Rep. 2018, 51, 280-289. [CrossRef] [PubMed]

45. Liu, S.J.; Dang, H.X.; Lim, D.A.; Feng, F.Y.; Maher, C.A. Long noncoding RNAs in cancer metastasis. Nat. Rev. Cancer 2021, 21, 446-460. [CrossRef] [PubMed]

46. Breiman, L. Random forests. Mach. Learn. 2001, 45, 5-32. [CrossRef] 